\title{
THE EFFECT OF CRYSTALLINITY ON THE THERMAL CONDUCTIVITY OF POLYMERS
}

${ }^{*}$ Noor S. Zaki ${ }^{1}$

\section{Tawfeeq W. Salih ${ }^{1}$}

1) Materials Engineering Department, College of Engineering, Mustansiriyah University, Baghdad, Iraq

\begin{abstract}
The aim of this research is investigating the direct effect of crystallinity of thermoplastic polymers on their thermal conductivity values. The study has included many materials, namely: polyoxymethylene (POM), highdensity polyethylene (HDPE), low-density polypropylene (LDPE), polypropylene (PP), polyamide (PA) and polyethylene terephthalate (PET). The degrees of crystallinity ranged from high-values (60-70\%), mid-values $(30-40 \%)$ and low-values (10-20\%). The work has done theoretically and experimentally. Theoretical work has used mathematical function extracted from reliable empirical relationships. The experimental work has included: manufacturing the specimens; specifying the cooling procedure in order to get the required degree of crystallinity; measuring the coefficient of thermal conductivity for different polymers at various conditions; and finally analyze the data and introduce the experiences obtained from the investigation. In general, theoretical values as well as experimental data were both behave similarly with respect to the variation of thermal conductivity with the degree of crystallinity. The results show that by increasing the degree of crystallinity the thermal conductivity of the polymer has increased by 10 $20 \%$.
\end{abstract}

Keywords: thermal conductivity, crystallinity, polymer, plastic, heat transfer

\section{Introduction}

Polymers have a variety of properties that depend on the field in which they are used, including physical, mechanical, and chemical properties [1]. The most useful classification of polymers for engineering purposes is based on their thermo-physical reaction. Polymers are known as thermoplastics or thermosets in this scheme. Thermoplastic polymers, as the name implies, melt and flow when subjected to heat and strain. Polyethylene, polystyrene, and nylon are examples of thermoplastic polymers. Crystallinity refers to the degree of structural order in a solid. In a crystalline polymer, the molecules are arranged in a regular, periodic manner. Synthetic polymers are either semicrystalline or amorphous. The degree of crystallinity has a big influence on physical and mechanical properties [2-4].

Thermal conductivity is one of the most important thermo-physical properties and has an essential impact for many applications, where polymers usually have much lower thermal conductivity than metals or ceramic materials, making them effective as thermal insulators [5]. A few studies have taken into account the linkage between the crystallinity and thermal conductivity of polymers [6-12]. For example, Zarandi M. et al (2012) [7] have studied the effect of crystallinity and irradiation on thermal

*Corresponding Author: ebma023@uomustansiriyah.edu.iq 
properties of LDPE under different cooling methods. The variation of thermal conductivity (k) of the samples show that when the cooling rate is increased, the percentage of crystallinity is decreased, and the thermal conductivity is decreased as well. Furthermore, Bai L. et al (2018) [10] have studied the effect of crystallinity and molecular chain orientation on the thermal conductivity of polymers. They used poly-L-lactide (PLLA), whose crystallinity and orientation can be adjusted in a wide range. It is found that below $\mathrm{Tg}$, the thermal conductivity of PLLA with different crystallinity increases with temperature and when the temperature is higher than $\mathrm{Tg}$, the thermal conductivity of PLLA with different crystallinity decreases remarkably. It is also demonstrated generally that the thermal conductivity of PLLA increases with the increasing of the crystallinity. These studies involved the crystallinity as an indirect parameter influences on the thermal property of polymer by describing morphological effects or thermal behaviors due to cooling process, chain orientation and crosslinking rather than focusing upon the direct relationship between the degree of crystallinity and the thermal conductivity, as it is proposed in this study. The materials used in the study (POM, HDPE, LDPE, PP, PA and PET) are thermoplastic materials have a range of values of degree of crystallinity.

\section{Theoretical Work}

Each chosen polymer has an interval of degree of crystallinity (Xc), as shown in Table 1.

Table 1. Degree of crystallinity for selected polymers $\lceil 1-3,13,14\rceil$

\begin{tabular}{cc} 
& {$[1-3,13,14]$} \\
\hline Polymer & Degree of crystallinity $(\mathbf{X c})$ \\
\hline POM & $0.2-0.8$ \\
HDPE & $0.2-0.8$ \\
LDPE & $0.1-0.5$ \\
PP & $0.1-0.5$ \\
PA & $0.1-0.3$ \\
PET & $0.1-0.3$ \\
\hline
\end{tabular}

\subsection{Thermal Conductivity}

A material's thermal conductivity is an indicator of its ability of the material to conduct heat. The coefficient of thermal conductivity $(\mathrm{k})$ is the express that defines the temperature gradient within this substance, making it an interesting parameter in heat transfer problems. Density, temperature, moisture, porosity, chain composition, degree of crystallinity, impurities molecule size and grain orientation are all variables that can influence a material's thermal conductivity [5]. The coefficient of thermal conductivity of polymers, in particular, behaves differently depending on whether the polymer is thermoplastic or thermosetting, and whether it is below glass transition temperature or above it [9]. The density has a strong effect on thermal conductivity, with higher density materials having higher thermal conductivity. Since increasing the crystallinity leads to an increase in polymer density. As a result, thermal conductivity is heavily influenced by the degree of crystallinity (Xc), where the thermal conductivity of crystalline part $(\mathrm{kc})$ is greater than that of amorphous one (ka) [6-10]. Simple mixing rule can be used to combine both crystalline and amorphous regions, as following $[8,11]$ :

$k=X c+(1-X c) K a$

Since $(\mathrm{ka}<\mathrm{kc})$ by a ratio of $(\mathrm{Z})$ which is less than 1 , hence the last equation is rewritten as;

$K=[(1-Z) X c+Z] K c$

For selected polymers, the value of $(Z)$ can be derived from references [1-12], as shown in Table 2. 
Table 2. Typical values of thermal conductivity for selected polymers $[1,12]$

\begin{tabular}{ccc} 
Polymer & $\begin{array}{c}\mathrm{k} \text { of crystalline } \\
\text { structure }(\mathrm{W} / \mathrm{m} . \mathrm{K})\end{array}$ & $\mathrm{Z}=\mathrm{ka} / \mathrm{kc}$ \\
\hline POM & 0.37 & 0.8 \\
HDPE & 0.52 & 0.7 \\
LDPE & 0.32 & 0.5 \\
PP & 0.22 & 0.8 \\
PA & 0.30 & 0.9 \\
PET & 0.19 & 0.9 \\
\hline
\end{tabular}

\section{Experimental Work}

The experimental works have done in the Department of Materials, College of Engineering, Mustansiriyah University. The DSC tests have done in the Laboratory of Packing Materials in Ministry of Industry and Minerals (MOIM). While, the thermal conductivity tests have done in the Laboratory of Materials Research in Ministry of Science and Technology (MOST).

\subsection{Materials}

The demanded thermoplastic materials were gathered as grains from the local market in this regard. Six thermoplastic materials have been collected: Polyoxymethylene (POM), High Density Polyethylene (HDPE), Low Density Polyethylene (LDPE), Polypropylene (PP), Polyamide (PA), and Polyethylene Terephthalate (PET).

\subsection{Manufacturing of the Specimens}

The work involved melting the grains in a specific oven brand JRAD, with a thermostat operating up to $260{ }^{\circ} \mathrm{C}$, to create a series of circular specimens with $40 \mathrm{~mm}$ diameter and 5 mm thickness (according to ASTM C518 - 04), as shown in Fig. (1) and (2). The time it took for the plastic grains to melt in the oven was between 20 and 25 minutes, starting from room temperature $\left(20-22^{\circ} \mathrm{C}\right)$ and at a steady rate of heat $\left(10^{\circ} \mathrm{C} / \mathrm{min}\right)$. The specimen was then cooled using various techniques to achieve varying cooling speeds and, as a result, different degrees of crystallinity. Cooling can be done in a shut-off oven (with a cooling rate of $1-2{ }^{\circ} \mathrm{C} / \mathrm{min}$ ), in still air (with a cooling rate of $3-5{ }^{\circ} \mathrm{C} / \mathrm{min}$ ), or in a fridge (with a cooling rate of $6-8{ }^{\circ} \mathrm{C} / \mathrm{min}$ ).

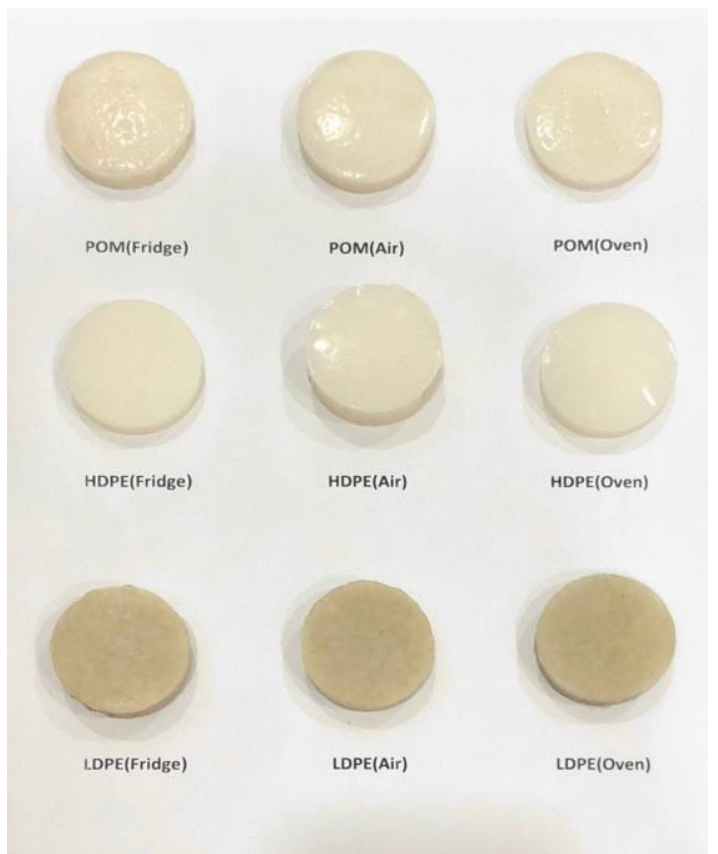

Figure 1. Shapes of POM, HDPE, LDPE specimens $(4 \mathrm{~cm}$ diameter and $0.5 \mathrm{~cm}$ thickness) for k-test

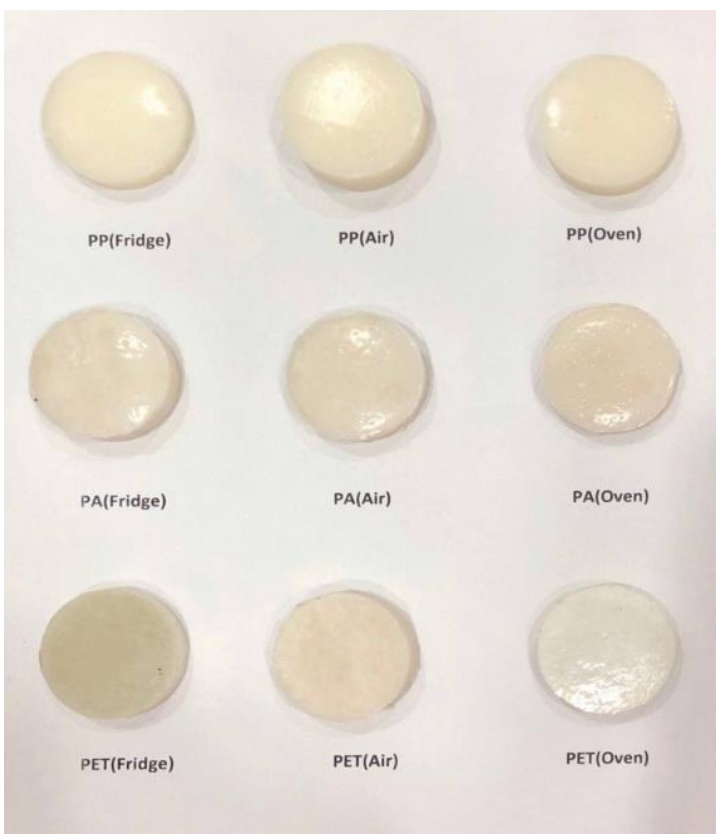

Figure 2. Shapes of PP, PA, PET specimens $(4 \mathrm{~cm}$ diameter and $0.5 \mathrm{~cm}$ thickness) for k-test. 


\subsection{Measurements}

\subsubsection{Degree of crystallinity}

Differential scanning calorimetry (DSC) is used in order to obtain the resultant degree of crystallinity for the selected polymers. A SHIMADZU analyzer (DSC-60) is used for this test, as shown in Fig. 3.

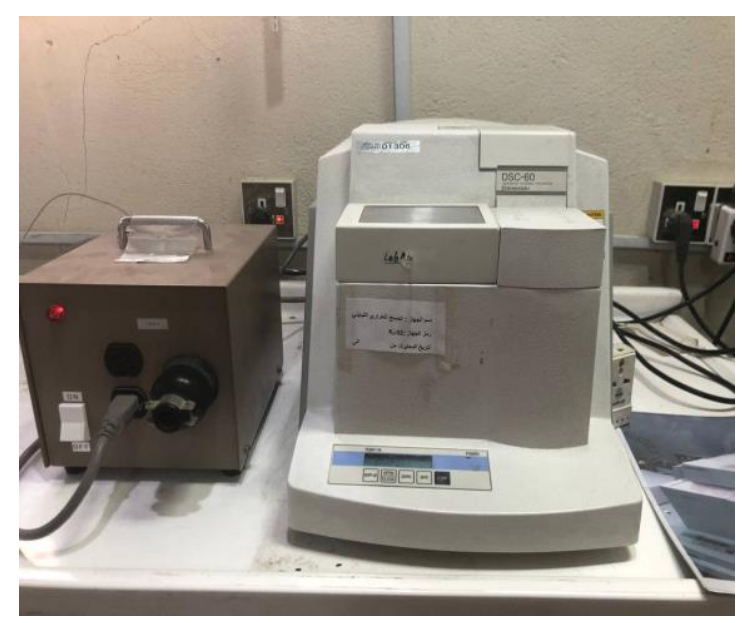

Figure 3. DSC analyzer type (SHIMADZU)

The procedure necessitates the use of small samples weighing between 2 and $10 \mathrm{mg}$ that are heated and melted in the DSC analyzer. The DSC approach is assumed that the amount of energy needed to heat the sample is measured and compared to the amount of energy required to heat a reference sample that does not undergo any phase transition in the range of interest $[15,16]$. For this purpose, some polymers were grounded into small samples with an average diameter of $2.36 \mathrm{~mm}$ or Mesh 8 (shown in Fig. 4 and 5) as required for standard DSC tests.

The common way of calculating polymer crystallinity is by measuring the enthalpy of fusion ( $\triangle \mathrm{Hf}$ ) by DSC and comparing it to the reference value of melting heat for fully crystalline polymer $(\Delta \mathrm{Hc})$, which is shown in Table 3.
Table 3. Melting heat (heat of fusion) for fully crystalline polymers [17-23]

\begin{tabular}{ll}
\hline Polymer & $\mathbf{\Delta H c}(\mathbf{J} / \mathbf{g})$ \\
\hline POM & 326 \\
HDPE & 202 \\
LDPE & 290 \\
PP & 207 \\
PA & 226 \\
PET & 136 \\
\hline
\end{tabular}

Hence, the degree of crystallinity $(\mathrm{Xc})$, is given by [1-3]:

$X c=\frac{\Delta H f}{\Delta H_{c}}$

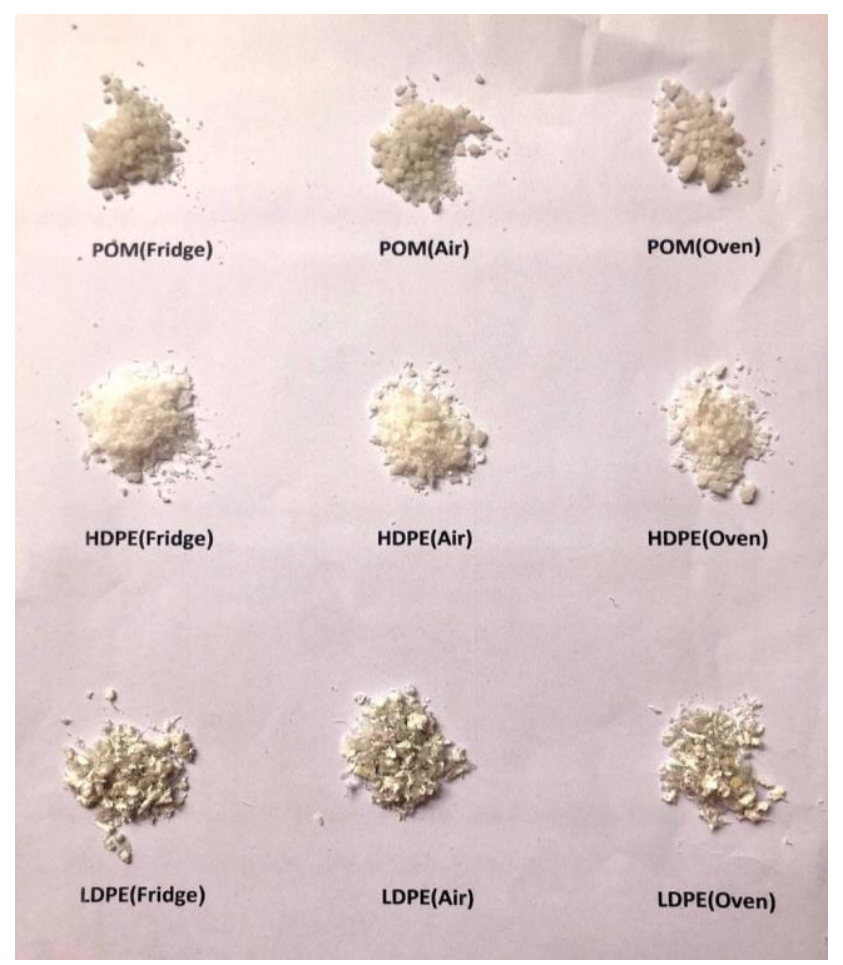

Figure 4. Samples prepared for DSC test of (POM, HDPE and LDPE) 


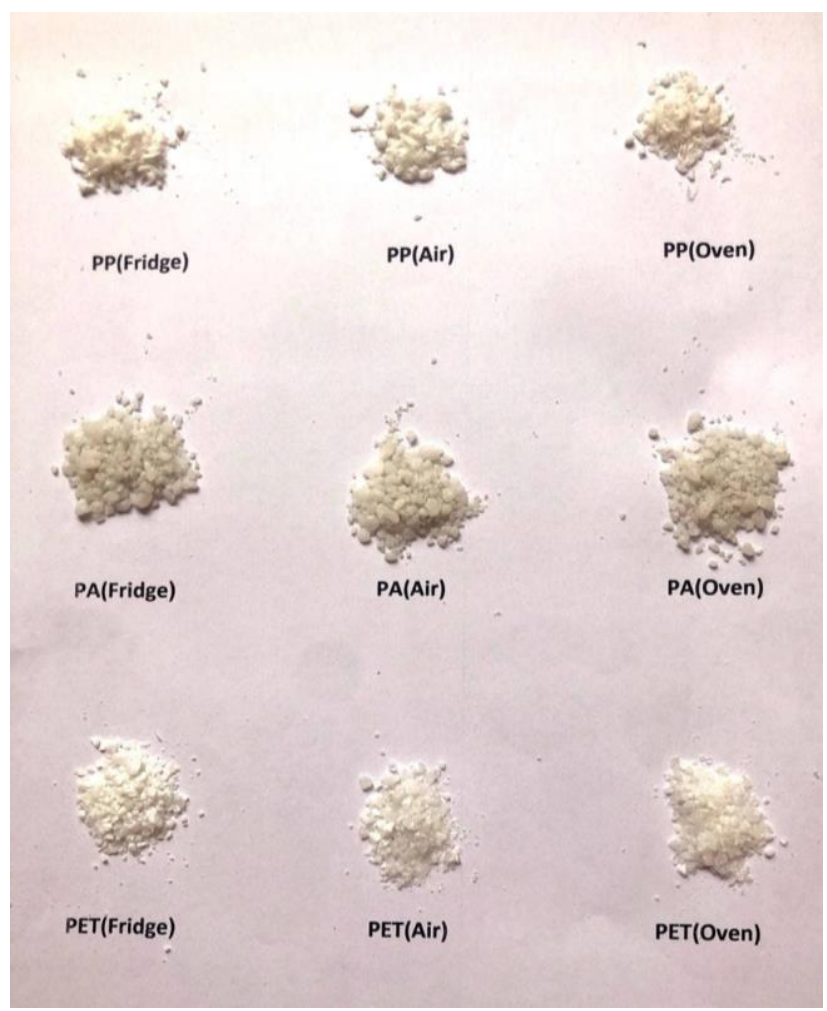

Figure 5. Samples prepared for DSC tests of (PP, PA and PET)

\subsubsection{Coefficient of thermal conductivity}

Lee discs apparatus is used to calculate the coefficient of thermal conductivity. The unit is made up of a chamber and a reader. As shown in Fig. 6, the chamber contains an electric heater and a collection of disks, with the specimen placed between the hot and cold discs. The electric circuit should be turned on before equilibrium is achieved. The reader used sensors attached to a thermometer to record the temperatures on the both sides of the specimen. The reported values have been compensated using a special equation designed specifically for this unit. The general equation that regulates heat transfer in solid materials, on the other hand, is based on a one-dimensional steady-state heat equation (Fourier equation), as [24]:

$Q=K A \frac{(T 1-T 2)}{t}$

Where;

$\mathrm{Q}=$ Heat Transfer $(\mathrm{W})$. $\mathrm{k}=$ thermal conductivity $(\mathrm{W} / \mathrm{m} \cdot \mathrm{K})$.

$\mathrm{A}=$ the specimen's cross-sectional area $\left(\mathrm{m}^{2}\right)$.

$\mathrm{t}=$ thickness of the specimen $(\mathrm{m})$.

$\mathrm{T} 1=$ surface point reading at the hot side of the specimen $(\mathrm{K})$.

$\mathrm{T} 2=$ surface point reading at the cold side of the specimen (K).

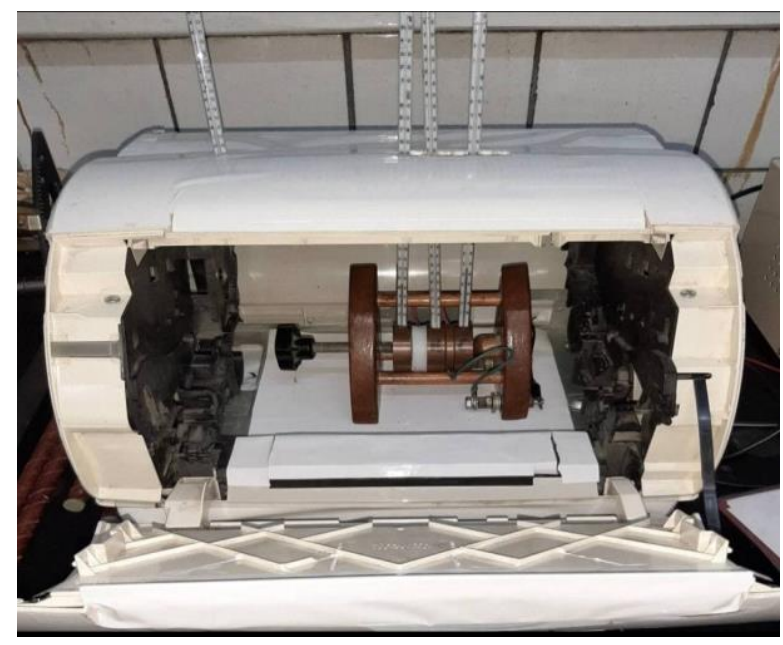

Figure 6. Lee discs apparatus

\section{Results and Discussions}

Since the rate of cooling was different from a sample to another for the same polymeric material, thus it gives a range of degrees of crystallinity. This leads to different thermal conductivity values based on the degree of crystallinity for each sample. The thermal conductivity has the highest value when the specimen was cooled in shut-off oven, then less value when it is cooled in still air and it has the least value when the cooling was in fridge, as shown in Fig. (7-12). This behavior occurs because the increasing of crystallinity means more packed structure, which acts as a shield, restricting the movement of conduction heat transfer from the material's surface to its core. The theoretical values differed than experimental values by less than $10 \%$, and they both behaved similarly. It is worth noting that the rise was linear for theoretical values but curvilinear for experimental values. In both cases, POM and 
HDPE show highest influence in the k-value due to wide range of degree of crystallinity. On the other hand, PA and PET show the least influence. In the overall, samples with the highest degree of crystallinity exhibit higher thermal conductivity by $10-20 \%$ as compared to samples with the lowest degree of crystallinity. This behavior can be recognized in the previous studies even though they have not referred to the percentage of variation between the degree of crystallinity and the coefficient of thermal conductivity. However, some errors are expected due to the variation in the reference values that extracted from different resources used in this study for both thermal conductivity and melting heat values. On the other hand, a percentage of error could be found in the experimental readings.

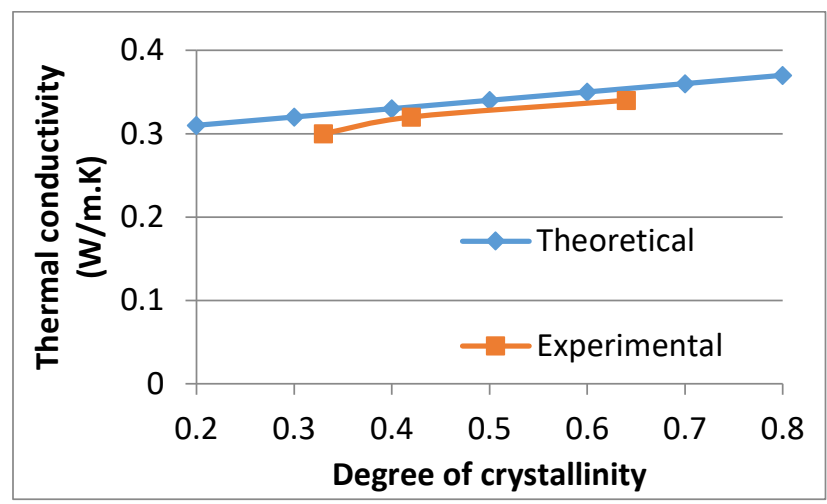

Figure 7. Variation of k-value with the degree of crystallinity for POM

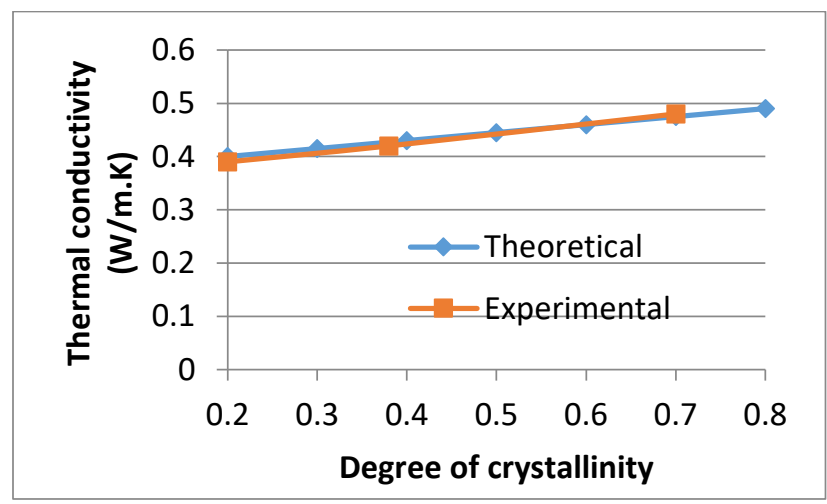

Figure 8. Variation of k-value with the degree of crystallinity for HDPE

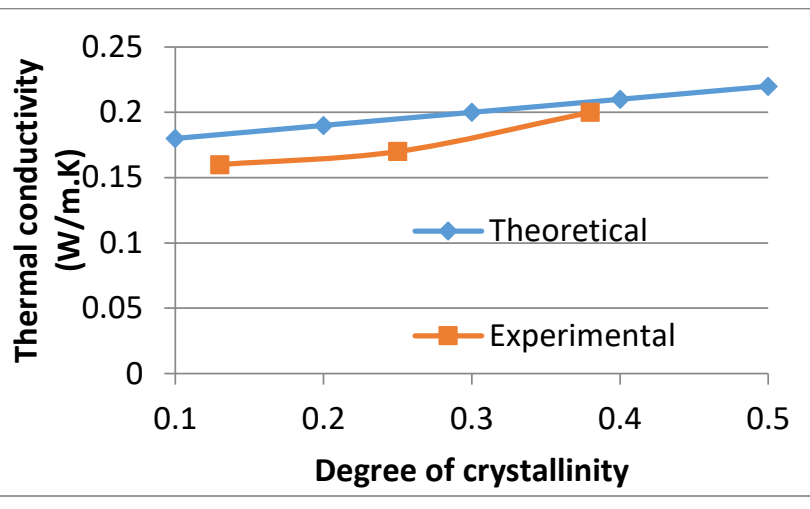

Figure 9. Variation of k-value with the degree of crystallinity for LDPE

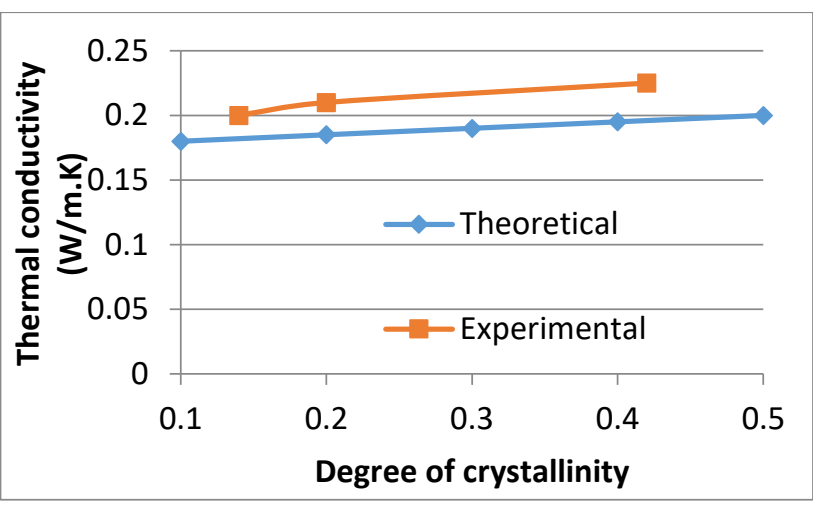

Figure 10. Variation of k-value with the degree of crystallinity for PP

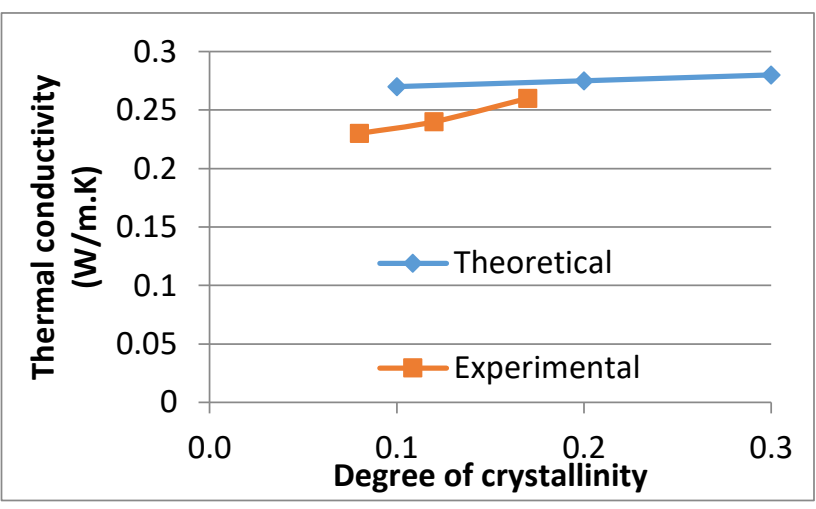

Figure 11. Variation of k-value with the degree of crystallinity for PA 


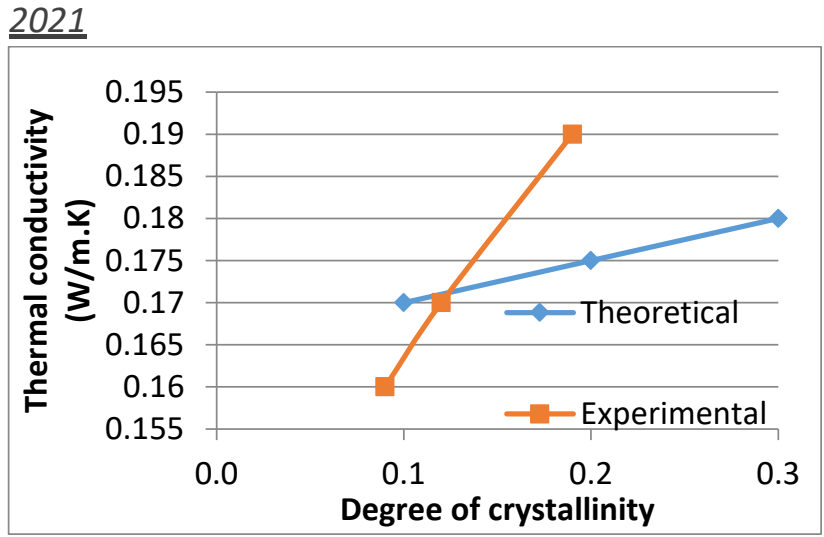

Figure 12. variation of k-value with the degree of crystallinity for PET

\section{Conclusions}

This study demonstrates for the direct influence of crystallinity on thermoplastic thermal conductivity, where the investigation concluded the following points:

- Theoretical values and experimental data behaved similarly, where the increasing in the degree of crystallinity leads to increase the k-value.

- The increasing in the value of thermal conductivity was $10-20 \%$ for samples that show the highest degree of crystallinity comparing to that show the lowest degree of crystallinity.

- However, these findings are limited to pure structure polymers (POM, HDPE, LDPE, PP, PA, and PET).

\section{Acknowledgements}

Authors are grateful to all support given by College of Engineering, University of Mustansiriyah for academically supporting.

\section{Conflict of Interest}

The authors confirm that the publication of this article causes no conflicts interest.

\section{References}

1. Krevelen D. and Nijenhuis K. (2009). "Properties of polymers". $4^{\text {th }}$ ed., Elsevier.
2. Ehrenstein G. (2001). "Polymeric materials: structure, properties,

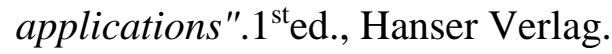

3. Cambridge (January 2005). "Crystallinity in polymers", University of Cambridge, DoITPoMS , UK.

4. Vieille B., Albouy W., Chevalier L. and Taleb L. (2013). About the influence of stamping on thermoplastic-based composites for aeronautical applications, Composites, Part B, Engineering, Vol.45, No.1, pp.821-834.

5. Tawfeeq W. (2021). "Insulation materials: fundamentals and applications". $1^{\text {sted., }}$ Mustansiriyah University.

6. Mtshali T., I. Krupab, A.S. Luyta. (2001). The effect of cross-linking on the thermal properties of LDPE/wax blends. Thermochimica Acta, Vol.380, No1, pp.4754, Elsevier.

7. Zarandi M., Hojjat A., Zahra-alsadat M., Farshid T., Ghazanfar M. (2012). Effect of crystallinity and irradiation on thermal properties and specific heat capacity of LDPE \& LDPE/EVA. Applied Radiation and Isotopes, Vol.70, No.1, pp.1-5, Elsevier.

8. Zinet M., Zakariaa R., M'hamed B., Shihe X., Patrick B. (2013). Thermophysical characterization and crystallization kinetics of semi-crystalline polymers. Journal of Modern Physics, Vol.4, pp.2837.

9. Dos Santos W., J.A. de Sousa, R. Gregorio Jr. (2013). Thermal conductivity behaviour of polymers around glass transition and crystalline melting temperatures. Polymer Testing, Elsevier, Vol.32, No.5, pp.987994.

10. Bai L., Xing Z., Rui-Ying B., ZhengYing L., Ming-Bo Y. and Wei Y. (2018). Effect of temperature, crystallinity and 
$\underline{\underline{2021}}$

molecular chain orientation on the thermal conductivity of polymers: a case study of PLLA. J. Mater Sci, Springer, Vol.53, pp.10543-10553.

11. Hongyu C., Valeriy G., Jian Y., Yunfeng Y., Wei L., Yan H., Libo D., Bin C. (2016). Thermal conductivity of polymer-based composites: Fundamentals and applications. Progress in Polymer Science, Vol.59, pp.41-85.

12. Zhang T., Wu X., Luo T. (2014). Polymer nanofibers with outstanding thermal conductivity and thermal stability: fundamental linkage between molecular characteristics and macroscopic thermal properties. J Phys Chem C, Vol.118. No.36, pp.21148-59.

13. Fried J. (2014). "Polymer science and technology". $3^{\text {rd }}$ ed., Prentice hall.

14. Ebewele O. (2000). "Polymer science and technology", CRC press.

15. Dean J. (1995). The analytical chemistry handbook. New York: McGraw Hill Inc., Vol.15, No.5, pp.1-15. ISBN 0-07016197-6.

16. Pungor E. (1995). "A practical guide to instrumental analysis". $1^{\text {st }}$ d., Boca Raton, pp. 181-191.

17. Woo M., Wong P., Tang Y., Triacca V., Gloor P., Hrymak A. (1995). Melting behavior and thermal properties of highdensity polyethylene. Polymer engineering and science, Vol. 35, No.2, pp.151-156.

18. Luiz H. Poley, Ana P. L. Siqueira, Marcelo G. da Silva, Helion Vargas. (2004). Photothermal characterization of lowdensity polyethylene food packages. Polímeros: Ciência e Tecnologia, vol. 14, No. 1, pp. 8-12.

19. Roger L. Blaine. (2002). "Thermal applications note: polymer heats of fusion".
Research Report No. TN048, TA Instruments, USA.

20. Howard W. Starkweather Jr., Paul Zoller, Glover A. Jones. (1983). The heat of fusion of poly (ethylene terephthalate). Wiley, Polymer physics, Vol.21, No. 2.

21. Lin Q., Yee A. (1997). Interactions of a liquid crystalline polymer with polycarbonate and poly (ethylene terephthalate). Journal of materials science, Vol.32, No.15, pp.3961-3970.

22. Pielichowska K. (April 2012). The influence of molecular weight on the properties of polyacetal/hydroxyapatite nanocomposites. Part 1. Microstructural analysis and phase transition studies. Journal of Polymer Research, Springer Vol. 19, pp.9775.

23. Kong Y., Hay J. (2002). The measurement of the crystallinity of polymers by DSC. Polymer, Elsevier, Vol.43, No.14, pp.3873-3878.

24. Holman J. (2010). "Heat transfer". 10 ed., McGraw-Hill Company. 